\title{
FACTORIZATION OF OPERATOR VALUED ENTIRE FUNCTIONS
}

\author{
BY MARVIN ROSENBLUM AND JAMES ROVNYAK ${ }^{1}$
}

Communicated by Irving Glicksberg, June 23, 1969

Let $W(z)$ be a complex valued entire function of exponential type with nonnegative values on the real axis. We call $W(z)$ factorable if

$$
W(z)=A^{*}(z) A(z)
$$

where $A(z)$ is an entire function whose restriction to the upper halfplane is an outer function. Here $A^{*}(z)=\bar{A}(\bar{z})$. Recall that an outer function in the upper half-plane is a function of the form

$$
f(z)=C \exp \left(\frac{1}{\pi i} \int_{-\infty}^{+\infty} \frac{1+t z}{t-z} \frac{\log k(t)}{1+t^{2}} d t\right), \quad y>0,
$$

where $C$ is a constant of absolute value $1, k(t) \geqq 0$ a.e. on $(-\infty, \infty)$, and $\left(1+t^{2}\right)^{-1} \log k(t) \in L^{1}(-\infty, \infty)$. Of necessity, $k(x)=\lim |f(x+i y)|$ a.e. where the limit is taken as $y$ decreases to zero. Therefore the restriction of an entire function $A(z)$ to the upper half-plane is an outer function if and only if $\left(1+t^{2}\right)^{-1} \log |A(t)| \in L^{1}(-\infty, \infty)$ and

$$
\log |A(z)|=\frac{y}{\pi} \int_{-\infty}^{+\infty} \frac{\log |A(t)|}{(t-x)^{2}+y^{2}} d t, \quad y>0 .
$$

The following facts are available from the classical theory of entire functions:

$\left(1^{\circ}\right)$ for $W(z)$ to be factorable, it is necessary and sufficient that

$$
\int_{-\infty}^{+\infty} \frac{\log +W(x)}{1+x^{2}} d x<\infty,
$$

$\left(2^{\circ}\right)$ if $W(z)$ is factorable, the factor $A(z)$ is determined to within a multiplicative constant of absolute value 1 ,

$\left(3^{\circ}\right)$ if $W(z)$ is factorable, say $W(z)=A^{*}(z) A(z)$ as above, and if $W(z)$ is of exponential type $\tau$, then $\exp \left(-\frac{1}{2} i \tau z\right) A(z)$ is of exponential type $\frac{1}{2} \tau$. See $[2$, p. 125], [3, p. 34], and [4, p. 437], where some original sources are cited. The purpose of this note is to communicate extensions of these results to operator valued entire functions.

\footnotetext{
1 Research supported by NSF Grant GP 8981.
} 
Let $\mathfrak{C}$ be a separable complex Hilbert space. By a vector or operator valued function we shall mean a function whose values are vectors in $\mathfrak{C}$ or bounded operators on $\mathfrak{C}$ respectively. Analyticity is defined in the weak sense. The bar of a bounded operator on $\mathcal{C}$ denotes its adjoint, and we use the notation $A^{*}(z)=\bar{A}(\bar{z})$ for operator as well as scalar valued entire functions. By $H_{\mathfrak{e}}^{2}$ we mean the Hardy space of vector valued analytic functions $f(z)$ defined for $y>0$ such that

$$
\|f\|^{2}=\lim _{y \rightarrow 0} \int_{-\infty}^{+\infty}\|f(x+i y)\|_{e}^{2} d x<\infty .
$$

If $\mathbb{B}$ is a closed subspace of $\mathfrak{C}, H_{\mathfrak{Q}}^{2}$ denotes the closed subspace of $H_{\mathfrak{e}}^{2}$ of functions with values in $B$. An operator valued analytic function $A(z)$ defined for $y>0$ is called outer if there exists a bounded scalar valued outer function $f(z)$ such that

(i) $B(z)=f(z) A(z)$ is bounded for $y>0$, and

(ii) the range of multiplication by $B(z)$ in $H_{\mathfrak{e}}^{2}$ is dense in a subspace of the form $H_{\mathfrak{B}}^{2}$.

In this case the closed subspace $B$ of $\mathfrak{C}$ implied in (ii) does not depend on the choice of $f(z)$, and we say that $A(z)$ acts in $B$. A vector valued entire function $f(z)$ is said to be of exponential type $\tau, \tau \geqq 0$, if for every vector $c$ in $\mathfrak{C}$ the scalar valued entire function $f_{c}(z)=\langle f(z), c\rangle_{\mathfrak{e}}$ is of exponential type $\tau$. An operator valued entire function $W(z)$ is said to be of exponential type if for every vector $c$ in $\mathfrak{C}$ the vector valued entire function $W(z) c$ is of exponential type $\tau(c)$ for some number $\tau(c) \geqq 0$.

Let $W(z)$ be an operator valued entire function of exponential type which has nonnegative values on the real axis. We call $W(z)$ factorable if

$$
W(z)=A^{*}(z) A(z)
$$

where $A(z)$ is an operator valued entire function whose restriction to the upper half-plane is an outer function. We can now state our main results. Let $I$ denote the identity operator on $\mathcal{C}$.

THEOREM 1. Let $W(z)$ be an operator valued entire function of exponential type which has nonnegative values on the real axis. Assume that there exists a scalar valued entire function $w(z)$ of exponential type such that

$$
W(x) \leqq w(x) I
$$

for all real $x$. If $w(z)$ is factorable, then so is $W(z)$. 
THEOREM 2. Let $W(z)$ be an operator valued entire function of exponential type which has nonnegative values on the real axis. For each $j=1,2$, let

$$
W(z)=A_{j}^{*}(z) A_{j}(z)
$$

where $A_{j}(z)$ is an operator valued entire function whose restriction to the upper half-plane is an outer function, and let this outer function act in the subspace $B_{j}$ of $\mathcal{C}$. Then

$$
A_{2}(z)=U A_{1}(z)
$$

where $U$ is a partially isometric operator on $\mathbb{C}$ with initial set $B_{1}$ and final set $\AA_{2}$.

THEOREM 3. Let $W(z)$ be an operator valued entire function of exponential type which has nonnegative values on the real axis. Assume that $W(z)$ admits a scalar dominant as in Theorem 1. Assume that the scalar dominant is factorable, and let

$$
W(z)=A^{*}(z) A(z)
$$

where $A(z)$ is an operator valued entire function whose restriction to the upper half-plane is an outer function. If for some vector $c$ in $\mathfrak{e}, W(z) c$ is of exponenital type $\tau(c), \tau(c) \geqq 0$, then $\exp \left(-\frac{1}{2} i \tau(c) z\right) A(z) c$ is of exponential type $\frac{1}{2} \tau(c)$.

Consider the case $\operatorname{dim} e<\infty$. Let $W(z)$ be an entire function of exponential type which has nonnegative values on the real axis. If

$$
\int_{-\infty}^{+\infty} \frac{\log ^{+}[\operatorname{tr} W(x)]}{1+x^{2}} d x<\infty,
$$

then $W(z)$ is factorable. For in Theorem 1 we may choose $w(z)$ $=\operatorname{tr} W(z)$.

Another corollary is valid for an arbitrary separable coefficient space e. Let $W(z)=C_{0}+C_{1} z+\cdots+C_{2 N} z^{2 N}$ be a polynomial with operator coefficients which has nonnegative values on the real axis. Then

$$
W(z)=A^{*}(z) A(z)
$$

where $A(z)=A_{0}+A_{1} z+\cdots+A_{N} z^{N}$ is a polynomial with operator coefficients whose restriction to the upper half-plane is an outer function.

Proofs of these results will appear elsewhere. Theorem 2 is a special 
case of a more general assertion concerning outer functions. It is deduced from [ 6 , Theorem 3 ]. In the case where $W(z)$ is bounded on the real axis, i.e. when we can choose $w(z)$ to be a positive constant, Theorems 1 and 3 are proved using a Hilbert space method originated by D. Lowdenslager [5] and developed by the first author [6]. The general cases of Theorems 1 and 3 are obtained from this special case by means of a theorem of A. Beurling and P. Malliavin [1].

\section{REFERENCES}

1. A. Beurling and P. Malliavin, On Fourier transforms of measures with compact support, Acta Math. 107 (1962), 291-302.

2. R. P. Boas, Jr., Entire functions, Academic Press, New York, 1954.

3. L. de Branges, Hilbert spaces of entire functions, Prentice-Hall, Englewood Cliffs, N. J., 1968.

4. B. Ja. Levin, Distribution of zeros of entire functions, Transl. Math. Monographs, vol. 5, Amer. Math. Soc., Providence, R. I., 1964.

5. D. Lowdenslager, On factoring matrix valued functions, Ann. of Math. (2) 78 (1963), 450-454.

6. M. Rosenblum, Vectorial Toeplitz operators and the Fejer-Riesz theorem, J. Math. Anal. Appl. 23 (1968), 139-147.

University of Virginia, Charlottesville, Virginia 22903 